Revue d'histoire de l'Amérique française

ZAS REVUE D.HISTOIRE DE L'AMÉRIQUE FRANÇAISE

\title{
Armand Lavergne : son entrée dans la vie publique
}

\section{Marc La Terreur}

Volume 17, numéro 1, juin 1963

URI : https://id.erudit.org/iderudit/302252ar

DOI : https://doi.org/10.7202/302252ar

Aller au sommaire du numéro

Éditeur(s)

Institut d'histoire de l'Amérique française

ISSN

0035-2357 (imprimé)

1492-1383 (numérique)

Découvrir la revue

Citer cet article

La Terreur, M. (1963). Armand Lavergne : son entrée dans la vie publique. Revue d'histoire de l'Amérique française, 17(1), 39-54.

https://doi.org/10.7202/302252ar d'utilisation que vous pouvez consulter en ligne.

https://apropos.erudit.org/fr/usagers/politique-dutilisation/ 


\section{ARMAND LAVERGNE : SON ENTRÉE DANS LA VIE PUBLIQUE *}

Armand Lavergne est demeuré, pour bien des gens, le prototype de l'orateur cocardier, capable d'enthousiasmer un auditoire par ses envolées chevaleresques et de confondre un adversaire par un mot d'esprit habilement décoché. Plusieurs de ses contemporains, à qui je demandais des souvenirs personnels, m'ont répondu avec conviction, voire avec une flamme dans le regard: "Armand Lavergne, il parlait ce gars-là !». Le renseignement s'avère plutôt mince; mais n'illustre-t-il pas, avec une redoutable éloquence, le sort réservé aux hommes qui confinent leur activité au domaine politique ? Lavergne, en effet, a été constamment mêlé à la vie publique: pouvait-il en être autrement? Je ne le crois pas.

Les frères de sa grand'mère, les fameux Pacaud, ont presque tous participé à l'insurrection de 1837; son grand-père, Joseph-Guillaume Barthe, a fait du journalisme de combat toute sa vie, dirigeant même un temps Le Canadien. Ce farouche libéral était animé d'un tel amour pour l'ancienne mère-patrie qu'il décida d'aller y séjourner pour y faire connaître le Canada. Il est permis de supposer que le petit-fils se délectait du Canada reconquis par la France, où Barthe exaltait son amour pour la France, et des Souvenirs d'un demi-siècle, où il flétrissait la conduite souvent odieuse des Anglais. De plus, quelques-uns des Pacaud vivaient en étroite intimité avec leur nièce, madame Lavergne; l'un d'eux, Charles, habita un temps chez les Lavergne et tenait le jeune Armand «suspendu à ses lèvres à l'écouter conter les exploits merveilleux de ces héros de légende qu'étaient pour [lui] les hommes de la rébellion ». ${ }^{1}$ Du côté maternel donc,

* Travail présenté à la Réunion générale de l'Institut d'Histoire de l'Amérique française, le 27 avril 1963.

1 Armand La Vergne, Trente ans de vie nationale (Montréal, Les Editions du Zodiaque, 1935, 228 pages), 47. 
on retrouve une atmosphère authentiquement patriotique qui a enveloppé son enfance. Pour ajouter à cet atavisme, son père, Joseph Lavergne, associé et ami intime de Laurier, participe à chaque lutte électorale et siège même à Ottawa de 1887 à 1896; sa maison constitue le centre de ce foyer de libéralisme actif qu'est Arthabaskaville entre 1875 et 1896. Evidemment, les sujets de conversation, chez les Lavergne, se ramènent toujours à la politique et, bien sûr, à la politique libérale. Aussi n'est-il pas étonnant de voir Armand, dès l'âge de six ou sept ans, monter sur un banc, sur une chaise, pour haranguer ses petits camarades en s'inspirant, il va de soi, des arguments entendus à la maison. On le voit même, raconte son cousin Renaud, se hasarder à prendre la parole au salon familial. «Je me rappelle encore qu'un jour, écrit-il, durant une période électorale, où la question de la pendaison de Riel avait été de nouveau mise sur le tapis, qu'Armand excité par son entourage nous servit sa petite protestation avec feu: ce qui fit verser des larmes de joie à sa mère. $\gg^{2}$

À l'âge de dix ans, il entre au Séminaire de Québec mais ne perd pas, pour autant, son attrait pour les événements d'actualité car, chaque soir, il cause avec l'abbé Mathieu, entièrement gagné aux idées libérales; de plus, Laurier ne passe jamais à Québec sans le rencontrer au parloir du Séminaire, ce qui devait certes rehausser le prestige du jeune Armand auprès de ses condisciples. Une lettre qu'il écrit à sa mère, le 30 avril 1892 il vient d'avoir douze ans - montre jusqu'à quel point il peut être imbu de politique. Je ne puis m'empêcher de citer partiellement cette lettre encadrée de noir, pour rappeler le décès d'Alexander Mackenzie, et qui demeure étonnante venant d'un enfant de douze ans.

Fais mes amitiés au Grand Honorable, Honnête Chef [c'est de Laurier qu'il s'agit]. Dis-lui que je demeure parmi la cannaille bon libéral, canadienfrançais et patriote et qu'il assomme Sir A. P. [Sir Adolphe P. Caron], qu'il empale Abbott [Sir John 207.

2 Louis-Renaud La Vergne, Mémoires, Manuscrit dactylographié: 206- 


\begin{abstract}
Abbott], fasse sauté Sir Hector [Langevin], et qu'il fasse dansé Sir John Thomson enfin toute la Cannaille. ... Si les bleus n'étaient pas si cannailles nous serions au pouvoir depuis longtemps.
\end{abstract}

Il y va ensuite de quelques rimes sur les Patriotes de 1837, sur Mercier et Laurier, puis il conclut, non sans détermination:

Oui, s'il faut reprendre les armes, le sang des patriotes coule dans les veines des Canadiens-français. Un jour viendra où la vertu triomphera et le vice fuira car Laurier nous aura fait recouvrir droits religieux et civils. ${ }^{3}$

Mais je termine l'évocation de ces souvenirs d'enfance qui laissent prévoir que l'enthousiasme de Lavergne l'entraînera, très tôt, dans l'action directe. C'est précisément cette entrée dans la politique que j'aimerais retracer en y distinguant deux phases: ce que j'appellerais une étape préliminaire, faite de lutte intérieure, qui s'étend de 1900 à janvier 1904 ; puis, l'entrée dans la carrière, c'est-à-dire son élection à la Chambre des Communes, le 16 f'évrier 1904.

C'est en 1896 qu'Armand Lavergne assiste, pour la première fois, à une assemblée politique ${ }^{4}$ : il entend Laurier proclamer les droits des minorités et promettre pleine justice aux Canadiens français du Manitoba. Le jeune rhétoricien croit en Laurier, sans raisonner, obnubilé par le grand homme qui représentait alors, dit-il, « la revanche de 1760 , la fin de toutes nos humiliations, de tous nos malheurs $\gg .{ }^{5}$ Il ne semble pas que le recul de Laurier sur la question des écoles du Manitoba, pas plus que ses protestations de fidélité à l'Angleterre en 1897, n'aient entamé le libéralisme exalté de son jeune ami. ${ }^{6}$ Mais la participation du Canada à la guerre sud-africaine et l'attitude de Bourassa devaient bouleverser le petit univers de Lavergne,

3 Armand Lavergne à sa mère, 30 avril 1892. Lettre conservée par Mlle Juliette Bussière, de Québec.

4 Armand Lavergne, discours du 5 février 1907. Débats de la Chambre des Communes, 1906-1907, II : col. 2566.

5 Armand La Vergne, Trente Ans ..., 69.

6 Ibid., 85. 
toujours sous le charme de Laurier, mais attiré par les prises de position du député de Labelle. Étudiant en droit à Québec, il distribue à ses confrères les discours de Bourassa ${ }^{7}$ et c'est en faveur de celui-ci que, peu à peu, la balance va pencher. Cette évolution n'est pas facilement perceptible, je l'avoue; on le représente comme le chef de file de ce mouvement de jeunesse à Québec ${ }^{8}$ et lui-même le laisse entendre, ${ }^{9}$ mais je n'en puis retrouver nulle part de preuve formelle. Mieux vaut, alors, suivre l'activité extra-académique de Lavergne. En janvier 1900, il appuie le candidat de Laurier contre un libéral indépendant dans le comté de Lotbinière. Aux côtés de Fitzpatrick et de Sir Henri Joly de Lotbinière, le jeune homme de dix-neuf ans fait des discours en français et en anglais. ${ }^{10}$ Il faut souligner qu'au même moment Bourassa, qui a démissionné pour protester contre l'envoi des contingents, se fait réélire dans Labelle. Le candidat ministériel est battu dans Lotbinière, mais un fait demeure: Lavergne, à cette date, approuve tacitement le geste de Laurier.

La presse ministérielle en vient à s'extasier devant l'effort canadien pour vaincre les Boers et à multiplier les protestations de loyalisme envers la Grande-Bretagne; Bourassa, lui, proteste énergiquement en Chambre et dans une série d'assemblées publiques. De son côté, Lavergne hésite de plus en plus, ${ }^{11}$ mais on le retrouve, aux élections générales d'octobre 1900, comme porte-parole du parti libéral dans une assemblée contradictoire tenue dans le comté de Rimouski. Il administre au représentant conservateur « une râclée formidable et termina au milieu des hourras pour M. Laurier et l'orateur », note un journal libéral. ${ }^{12}$

En 1901, Le Soleil ouvre les hostilités contre Bourassa; toutefois, Lavergne est un familier de la maison d'Ernest Pacaud, où sa mère séjourne régulièrement et où lui-même est fêté à l'occasion de son vingt et unième anniversaire de naissance. ${ }^{13}$

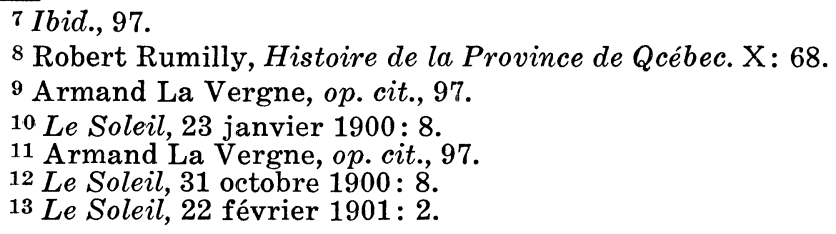


Or Pacaud, ennemi acharné de Bourassa, ${ }^{14}$ ne cache pas ses sentiments anglophiles et l'on va jusqu'à dire que «c'est un fait connu, à Québec, qu'on ne parle presque pas français dans la maison $d u$ directeur du SOLEIL $\gg .^{15}$ Cela n'empêche pas Lavergne de mêler sa voix à celles de vieux orateurs québecois, lors d'une grande assemblée tenue pour promouvoir la célébration de la St-Jean-Baptiste: il glorifie le drapeau de Carillon et le drapeau de la vieille France puis, en se faisant applaudir, il proclame la force des Canadiens français ( $\ldots$... si l'on nous menace, nous savons nous faire respecter comme lorsqu'on nous aime nous faire aimer $\gg) .{ }^{16}$

Au cours de cette même année 1901, cependant, l'attitude de notre jeune homme va évoluer: cette évolution et ses causes ne se laissent pas aisément circonscrire et l'absence de documents nous réduit à de pures conjectures. Les échos mondains nous le montrent, en juillet, donnant le bras à une dame de la suite de lord Minto, lequel visite La Malbaie: ${ }^{17}$ en septembre, l'accent presque anti-français imprimé aux réceptions faites au duc et à la duchesse d'York le révolte sûrement. ${ }^{18}$ Néanmoins, ses relations continuent d'être bonnes avec Fitzpatrick puisque, le 18 octobre, celui-ci prête un vaisseau du gouvernement aux étudiants en droit dont Lavergne est le président. ${ }^{19}$ Mais, dans une élection pour le comté de Québec où deux libéraux se présentent, on voit Armand Lavergne, le 27 octobre, prendre parti contre Fitzpatrick qui supporte, évidemment, le candidat parentiste. ${ }^{20}$ Le fossé s'élargit: moins d'un mois plus tard, il se rend à Montréal représenter les étudiants de Québec et il couvre Turgeon d'éloges dans le compte rendu du banquet qu'il écrit pour Le Soleil, ${ }^{21}$ allant même jusqu'à écrire que Turgeon «avait été sacré chef de la jeune génération sur laquelle le pays

14 Pacaud à Laurier, 28 décembre 1899, Papiers Laurier, A.P.C., M.G. 26, G 1 (a), vol. 135 : 40399-40402.

15 Les Débats, 31 août 1902: 1.

16 L'Evénement, 19 juin 1901: 2.

17 Le Soleil, 12 juillet 1901: 1.

18 Armand Lavergne, Lettre ouverte à L'Evénement, 14 mars 1902: 3.

19 Le Soleil, 18 octobre 1901: 8.

20 Le Soleil, 28 octobre 1901: 1.

21 Le Soleil, 23 novembre 1901: 1. 
compte pour l'avenir ».22 Vers la même date, Lavergne «est prêt, si une occasion s'en présente, à jeter toute la population anglaise dans le Saint-Laurent» et il «refuse systématiquement de parler ou d'étudier l'anglais ${ }^{23}$ ce qui désole Laurier. C'est donc à un quelconque moment de l'automne 1901 qu'Armand Lavergne s'est définitivement dégagé de l'atmosphère bassement impérialiste qui sévissait dans les milieux ministériels de Québec. De janvier à mai 1902, il est sérieusement atteint de la picote, séjourne plus d'un mois à l'hôpital, passe quelques mois de convalescence à Arthabaskaville et à Montréal, ${ }^{24}$ mais, dès le mois de mars, il est suffisamment rétabli pour prendre une position très nette sur le sujet qui nous intéresse.

Le 6 mars, un groupe de jeunes Québecois a constitué une association patriotique. Ils s'appellent « Les Revendicateurs des Droits et des Prérogatives de la langue française dans la Province de Québec », s'engagent à toujours parler français et à le faire parler à leurs amis, à purifier leur langue et à faire respecter intégralement le bilinguisme dans la province et la cité de Québec. ${ }^{25}$ Le Soleil, avec force arguments spécieux, condamne cette "petite campagne d'anglophobie ${ }^{26}$ mais «tout le pays a les yeux»sur ces trois cents jeunes gens, écrit un rédacteur des Débats. ${ }^{27}$ Armand Lavergne, indigné de «l'aplatissement et de la lâcheté »du Soleil, écrit une lettre virulente qu'il envoie à L'Événement, journal conservateur. ${ }^{28}$ Il feint de croire que Pacaud, fils de patriote, ne peut être l'auteur de l'article du Soleil qui condamnait ce mouvement de jeunesse, mais il tombe à bras raccourcis sur ces « gens sérieux ... les quémandeurs qui attendent une place ou un contrat du gouvernement, les engraissés à la crèche, les rebus et ceux qui . . . ont peur ... qu'on ne les trouble dans leurs jouissances plus ou

22 Le Soleil, 25 novembre 1901: 1.

23 Laurier à Mme Joseph Lavergne, 29 novembre 1901, lettre citée par Rumilly, Histoire ..., X: 67.

24 Joseph Lavergne à Laurier, 23 février 1902, Papiers Laurier, vol. 225 : $63070-071$.

25 Texte de ces résolutions dans L'Evénement, 7 mars 1902: 4.

26 Le Soleil, 10 mars 1902: 1. 1902: 2 .

${ }^{27}$ Louis Rabillon, Ça va très bien, merci !, dans Les Débats, 16 mars

28 Armand Lavergne, Lettre ouverte à L'Evénement, 14 mars 1902: 3. 
moins légitimes »... tous ceux-là, enfin, qui ont réveillé le sentiment des jeunes «saturés, écœurés》 des bassesses des «gens sérieux ». On retrouve, dans cette longue lettre, les accents violents et les protestations enflammées caractéristiques de Lavergne: «Nous sommes ici chez nous, dans la Nouvelle-France, et nous y resterons à combattre le combat de nos pères, la lutte de nos fiers aïeux.» Armand Lavergne vient de rompre les ponts : il interrompt sa convalescence pour descendre à Québec ${ }^{29}$ applaudir sans doute à la motion anti-impérialiste de J. A. Lane; mais celui-ci retire sa motion. Qu'à cela ne tienne, le grand pas est franchi et la grande assemblée nationaliste de Drummondville, le 19 juin 1902, compte, parmi ses orateurs, Henri Bourassa, Dominique Monet et Armand Lavergne: on y fait voter des résolutions qui proclament «l'autonomie du peuple canadien » et qui approuvent la position de Laurier qui, prudemment, a reculé sur la question de l'impérialisme. ${ }^{30}$ Lavergne semble ensuite se consacrer entièrement à ses études; il lui faut «être prêt... à soutenir l'honneur des étudiants qui se sont mêlés de politique pendant leur cours », écrit-il à Mgr Mathieu. ${ }^{31}$ Quoi qu'il en soit, la Ligue Nationaliste est fondée en mars 1903, Lavergne est reçu avocat en juillet et commence à pratiquer à Québec en octobre. À cette date, il semble faire bon ménage avec Le Soleil qui lui adresse louanges et vœux de succès tandis que Lavergne y insère chaque jour l'annonce de son bureau d'avocat. Mais il faut dire que Pacaud, très malade, doit se désintéresser de son journal qui tombe graduellement sous la coupe de Choquette, très hostile à Parent, que les nationalistes, non plus, n'aiment pas. Olivar Asselin, à qui Laurier a demandé un mémoire sur la situation de la colonisation, ${ }^{32}$ lui dit «ce que la jeunesse pense de la politique de M. Parent ».33 Il n'est pas étonnant, alors, de voir Lavergne, en novembre, démissionner comme ministre dans le Parlement modèle de Québec « pour pou-

29 L'Union des Cantons de l'Est, 21 mars 1902: 2.

30 Texte de ces résolutions dans L'Union des Cantons de l'Est, 20 juin 1902: 2; excellent compte rendu dans La Presse, 21 juin 1902: 29.

31 Lavergne à Mgr Mathieu, 11 mai 1902, ASQ, Université 5, no 98.

32 Olivar Asselin à Laurier, 16 septembre 1903, Papiers Laurier, vol. $280: 76818$.

${ }^{33}$ Le même au même, ibid., 76833. 
voir tomber à son goût sur M. Parent $»^{34}$ qu'il appellera, un peu plus tard, «ce maudit homme $»^{\mathbf{3 5}}$. Si l'on fait le point, en décembre 1903, on constate qu'à la vérité « un grand mouvement politique est à l'horizon $\gg^{36}$, mouvement qui reçoit sa consécration au Manège Militaire de Québec, le 8 décembre, devant 5 à 6,000 personnes. Bourassa, accompagné d'Asselin et de Lavergne, expose le programme de la Ligue, ajoutant au thème de l'anti-impérialisme un article fondamental: le développement des richesses naturelles du Québec au profit de ses habitants. Puis, devant une phalange de chefs libéraux - plusieurs ont pris place sur l'estrade - Bourassa attaque le gouvernement de la province. Le Soleil, qui jugeait auparavant ce programme en pleine conformité avec la doctrine du parti libéral, ${ }^{37}$ se verra obligé de dire à Bourassa «animé de bonnes intentions... [qu'] un plus minutieux examen le convaincra qu'il est plus facile d'écrire une thèse sur le papier que de résoudre ces problèmes d'administration pratique $»^{38}$ et l'honorable Jules Tessier avouera qu'il ne pensait pas y entendre « une pareille avalanche de propos malveillants contre [ses] chefs $\gg .{ }^{39}$

Bourassa fait parvenir à Laurier le programme de la Ligue en soulignant que les idées personnelles de ces jeunes «sont essentiellement canadiennes $\gg .{ }^{40}$ Vers la même époque, Ernest Roy, député de Montmagny à la législature provinciale, dispensateur dans le même comté du patronage fédéral, ${ }^{41}$ très sympathique aux idées nouvelles - il a pris la parole à l'assemblée du $8-{ }^{42}$ convaine Lavergne de venir s'installer à Montmagny en lui promettant de l'aider. ${ }^{43}$ Cette promesse revêt un sens par-

${ }^{34}$ L'Evénement, 2 février 1904: 2.

35 Lavergne à Rodolphe Boudreau, 15 septembre 1904, Papiers Laurier, vol. 125: 37407a.

36 Le Combat, 8 novembre 1903: 8.

37 Le Soleil, 27 novembre 1903: 11.

38 Le Soleil, 11 décembre 1903: 1.

39 Le Combat, 13 décembre 1903: 8. 8027.

40 Bourassa à Laurier, 25 décembre 1903, Papiers Laurier, vol 296 : 76965 .

41 Laurier à Ernest Roy, 15 septembre 1903, Papiers Laurier, vol. 281: 81455 .

42 Le Soleil, 9 décembre 1903: 5.

43 Lavergne à Laurier, 23 janvier 1904, Papiers Laurier, vol. 301 : 
ticulier lorsque l'on sait que le siège de Montmagny aux Communes est vacant depuis quatre mois et que des élections partielles, à défaut d'élections générales, devront être tenues dans un avenir prochain.

Dès le début de janvier, Laurier a décidé de ne pas dissoudre le Parlement: il lui faut donc combler les vacances. Il s'enquiert auprès d'Ernest Roy afin de savoir si les libéraux de Montmagny ont un candidat en vue. Nous en avons une pléiade, ${ }^{44}$ répond Roy, qui en énumère sept d'importance inégale: Savard, que la rumeur avait déjà désigné,45 Cyrias Roy, protonotaire et cousin d'Ernest, le jeune Armand Lavergne et quatre autres dont le juge Philippe-Auguste Choquette. Les réactions de ce dernier sont plutôt amusantes. Le 13 janvier, il écrit à Laurier pour lui dire qu'il est «presque décidé $»^{46}$ à poser sa candidature. Le premier ministre lui répond derechef: "Vous savez parfaitement que, quant à moi, je serais ravi de vous voir revenir dans la politique. ${ }^{47}$ Le 14 , Choquette fait parvenir à Laurier une liasse de lettres provenant de personnages importants du comté de Montmagny qui favorisent Lavergne. ${ }^{48}$ L'un de ces correspondants, le docteur Paradis, dit même à Choquette qu' «Armand Lavergne est justement l'homme qui tiendra la place chaude pour [lui] $\gg,{ }^{49}$ ce qui supposerait un calcul: Lavergne conserverait le comté pour le céder à Choquette à sa descente du banc. Choquette hésite, car, le 18, il mande à Laurier que, s'il «n'y va pas », Lavergne «sera un des meilleurs candidats ${ }^{50}$ Malgré ses tergiversations, Choquette demeure sur

\footnotetext{
80823-29.

44 Ernest Roy à Laurier, 7 janvier 1904, Papiers Laurier, vol. 298:

45 Les Débats, 20 septembre 1903: 4. 81024.

46 Choquette à Laurier, 13 janvier 1904, Papiers Laurier, vol. 299 : 81027.

47 Laurier à Choquette, 14 janvier 1904, Papiers Laurier, vol. 299 :

48 Lettres conservées dans les Papiers Laurier, vol. 299: 81034-81044. 81035.

49 Paradis à Choquette, 13 janvier 1904, Papiers Laurier, vol. 299 : 81257 .

50 Choquette à Laurier, 18 janvier 1904, Papiers Laurier, vol. 300 :
} 
le banc, mais fait jouer toute son influence en faveur de Lavergne ${ }^{51}$; dans son volume de souvenirs, Choquette affirme qu'il a agi de cette façon à la demande du juge Lavergne, père d'Armand, qui lui a laissé entendre que «cela ferait plaisir à Laurier $\gg .52$

Quels qu'en soient les motifs, cet appui était loin d'être négligeable et surtout loin d'être superflu, parce que de nombreuses et fortes oppositions se dessinaient, à l'intérieur du parti libéral, contre le jeune avocat. Le plus dangereux de ces adversaires demeurait Charles Fitzpatrick qui favorisait Cyrias Roy. Fitzpatrick, bras droit de Laurier dans la région de Québec, possédait plusieurs raisons de ne pas aimer Lavergne: l'on se souvient que ce dernier avait combattu le candidat parentiste dans le comté de Québec et Fitzpatrick avait alors voulu «le faire assommer $\gg{ }^{53} \mathrm{Ami}$ et associé légal de Parent, Fitzpatrick a réussi à circonvenir Ernest Roy. ${ }^{54}$ Voilà donc Lavergne privé de l'appui du député provincial qui l'a «sollicité 》de venir se présenter dans le comté. «Je le savais entre les mains de Fitzpatrick, écrit-il à Laurier dans un mouvement de colère, mais pas assez pour trahir sa parole»; et il ajoute: «pas plus que vous, je ne sais me contenir devant la duplicité et l'hypocrisie $\gg .55$

Toutefois, Lavergne ne manquera pas d'alliés: en plus de pouvoir compter sur Choquette (du moment qu'il ne se présente pas, il va nécessairement se ranger dans le camp opposé à celui de Fitzpatrick et Parent), il recevra l'assistance de son influente famille. Tout d'abord, son oncle Louis, député d'Arthabaska et whip du parti libéral pour la province, possédant des attaches dans le comté, consacre quatre jours « à passer [son neveu] de paroisse en paroisse » avant la convention, dit

51 Armand La Vergne, Trente Ans ..., 101, n. 1.

52 P.-A. Choquette, Un demi-siècle de vie politique (Montréal, Beauchemin, 1936, 352 p.), 147.

53 Joseph Lavergne à Laurier, 30 janvier 1904, Papiers Laurier, vol. 302: 81798 .

54 Laurier à Ernest Roy, 27 janvier 1904, Papiers Laurier, vol. 302 : 81720 : «Vous m'avez dit que Cyrias Roy était le meilleur candidat et celui qui emporterait la convention. 》 81454-57.

55 Lavergne à Laurier, 23 janvier 1904, Papiers Laurier, vol. 301: 
un observateur, ${ }^{56}$ quinze jours à visiter "tous les délégués choisis », écrit son fils Renaud. ${ }^{57}$ Puis, Louis-Philippe Brodeur prête main-forte à la convention parce que Lavergne «n'a pas voulu se fier à M. Fitzpatrick ».58 Mais un facteur important subsiste: sir Wilfrid Laurier, le grand arbitre de tous les conflits qui surviennent dans le camp libéral. Va-t-il donner son appui à Lavergne, dont le libéralisme s'éloigne un peu de l'orthodoxie mais auquel il demeure très attaché ? Ou bien va-t-il se ranger à l'avis de son ministre Fitzpatrick ?

La famille Lavergne fait, au sens strict, le siège de Laurier. Armand lui représente qu'il est «malgré tout, un bon soldat »;59 son père, l'ami de Laurier, multiplie les lettres. «Armand est un peu indépendant, mais il vient de faire une profession de foi libérale... Il a de l'avenir et peut devenir un homme tandis que Roy sera toujours une médiocrité... ${ }^{60}$ Par son représentant Fitzpatrick ton gouvernement fait un jeu de duplicité qui n'est pas édifiant. Armand ne serait pas allé à Montmagny s'il avait cru avoir l'hostilité déclaré [sic] du gouvernement... toi de ton coté [sic] tu ne nous auras pas traités comme de vieux amis. Je croyais qu'à chance égale tu aimais autant Armand que Cyrias Roy...» Les délégués seront cabalés d'avance, « mais ce sera une injustice et une malhonneteté [sic] qui ne peut que dégouter [sic] bien des amis $\gg .{ }^{61}$ L'oncle Louis, pour sa part, demande carrément à Laurier s'il aimerait « voir Armand choisi comme le candidat du gouvernement $\gg .{ }^{62}$

Mais le premier ministre, fidèle à son principe, laisse à la convention le choix du candidat, même s'il avoue à Louis «qu'à

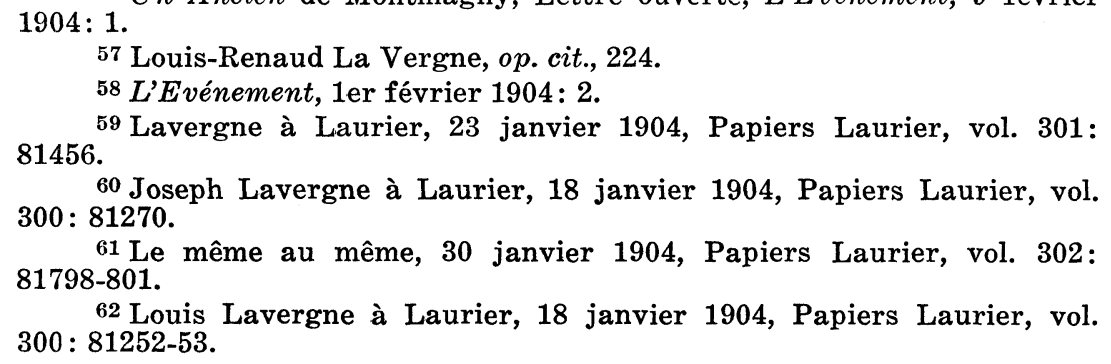

57 Louis-Renaud La Vergne, op. cit., 224.

58 L'Evénement, 1er février 1904: 2. 81456.

59 Lavergne à Laurier, 23 janvier 1904, Papiers Laurier, vol. 301: $300: 81270$. 81798-801.

61 Le même au même, 30 janvier 1904, Papiers Laurier, vol. 302 :

62 Louis Lavergne à Laurier, 18 janvier 1904, Papiers Laurier, vol. $300: 81252-53$.

56 Un Ancien de Montmagny, Lettre ouverte, L'Evénement, 9 février 
cause de nos liens d'amitié, je serais très heureux de voir Armand choisi, mais j'ai toujours considéré, ajoute-t-il, que ce serait un miracle qu'un enfant comme lui fût choisi candidat. ${ }^{63}$ Louis, tenace, lui souffle: "Vous pourriez peut-être dire que si Armand était choisi, ça vous ferait plaisir $\gg,{ }^{64}$ mais Laurier demeure inflexible et, à la veille de la convention, il télégraphie: «Choix du candidat n'est pas entre mes mains, mais entre les mains des électeurs.» ${ }^{65}$ Néanmoins, il devient évident que le jeune aspirant gagne du terrain auprès des délégués. Question de fait, ceux-ci le choisissent, dans la proportion de deux contre un, le ler février, en présence de Bourassa, Fitzpatrick et Brodeur. L'avocat de vingt-trois ans, «pâle, l'émotion étreignant sa voix $\gg,{ }^{66}$ les remercie. On parle de convention arrangée, de candidat imposé ... « un tout jeune homme... un aventurier politique ${ }^{67}$... qui ne connaît rien au comté de Montmagny ${ }^{68}$ Qu'importe ! Lavergne est choisi et il faut batailler ferme car les conservateurs présentent un candidat d'envergure, Maurice Rousseau, maire de Montmagny, et Savard, évincé à la convention, demeure sur les rangs.

En premier lieu, on doit amener Savard à se désister, car la division du vote libéral permettrait à Rousseau de remporter la victoire. ${ }^{69}$ Lavergne s'en ouvre à Laurier et lui demande d' «ôter Savard de dans [sic] notre chemin ${ }^{70}{ }^{70}$ lequel Savard «serait prêt à s'effacer sur la promesse d'une position », renchérit Choquette. ${ }^{71}$ En fin de compte il incombe à Fitzpatrick,

63 Laurier à Louis Lavergne, 20 janvier 1904, Papiers Laurier, vol. $300: 81254$.

64 Louis Lavergne à Laurier, 23 janvier 1904, Papiers Laurier, vol. 301: 81449 . 81780.

65 Laurier à Lavergne, 30 janvier 1904, Papiers Laurier, vol. 302 :

66 Le Soleil, 2 février 1904: 2.

${ }^{67}$ Un Electeur de Cap St-Ignace, Lettre ouverte à L'Evénement, 5 février 1904: 2.

68 Un Ancien de Montmagny, Lettre ouverte à L'Evénement, 9 février $1904: 1$.

69 James D. Stewart à Choquette, 6 février 1904, Papiers Laurier, vol. 303: 82105 .

70 Lavergne à Laurier, 3 février 1904, Papiers Laurier, vol. 303: 81991. 82059 .

71 Choquette à Laurier, 5 février 1904, Papiers Laurier, vol. 303 : 
《la figure contractée de grimaces », note L'Événement, ${ }^{72}$ d'aller faire pression auprès de Savard et tout semble indiquer qu'on lui ait alors promis une compensation : ${ }^{73}$ toujours est-il qu'un télégramme de Laurier à Savard amène le candidat libéral indépendant à s'effacer devant le jeune avocat régulièrement choisi. ${ }^{74}$ A la mise en nomination, le parti libéral présente donc, extérieurement du moins, un front uni. Je dis «extérieurement» parce que, à ce stade, il me paraît important de préciser la position de Lavergne au sein du parti ministériel.

Aux yeux de Laurier, le jeune Armand est un «disciple » de Bourassa et le premier ministre n'hésite pas à écrire au député de Labelle que ce «disciple» sera «another thorn in the flesh ». ${ }^{75}$ D'ailleurs, il tente un effort auprès de Lavergne qui, pour assurer son élection, lui a indiqué certaines faveurs qui pourraient être octroyées à des gens du comté ; ${ }^{76}$ Laurier l'assure de son entière collaboration mais ne manque pas l'occasion d'essayer de le ramener dans le droit chemin. "Avant de terminer, lui dit-il, permets-moi de te donner le conseil d'un vieil ami. Prends ta position franchement dans les rangs du parti auquel tu appartiens par tradition, par principe et par conviction. Tu as fait un peu jusqu'à présent le métier de frondeur, mais tu ne regretteras pas plus tard, j'en suis sûr, si à cette heure, décisive dans ta carrière, tu fais en sorte que ceux qui te veulent du bien ne s'entendent pas dire à chaque instant que ta loyauté au parti laisse à désirer. $\gg^{77}$ Pourquoi ces réticences ? A cause des antécédents nationalistes de Lavergne sans doute, mais peutêtre également à cause de son attitude indépendante devant les organisateurs du parti: ils lui ont demandé de signer un papier par lequel il s'engageait à approuver les actes du cabinet Laurier

72 L'Evénement, 11 février 1904: 1. 88432-35.

73 Lavergne à Laurier, 27 juillet 1904, Papiers Laurier, vol. 330 :

74 Le Soleil, 10 février 1904 : 4. 82032.

75 Laurier à Bourassa, 5 février 1904, Papiers Laurier, vol. 303 : 81991-95.

${ }^{76}$ Lavergne à Laurier, 3 février 1904, Papiers Laurier, vol. 303 : 81997.

77 Laurier à Lavergne, 6 février 1904, Papiers Laurier, vol. 303 : 
et le candidat a mis le dit papier dans sa poche, au risque de se voir privé des subsides ordinaires. ${ }^{78}$

Malgré tout, les ministériels lui prêtent leur appui entier. Ils vantent les bienfaits qu'a apportés le gouvernement Laurier et insistent sur la construction toute prochaine du Grand-Tronc Pacifique. Rodolphe Lemieux et Louis-Philippe Brodeur louangent le parti mais Bourassa et Lavergne, eux, ne se gênent pas pour dénoncer l'impérialisme ${ }^{79}$ et même, dit-on, ${ }^{80}$ pour faire appel aux sentiments des Canadiens français. La campagne est menée à vive allure, malgré le froid intense, incapable de calmer les esprits échauffés. ${ }^{81}$ Les conservateurs reprochent à Lavergne sa jeunesse: Le Soleil rétorque que Barthe, son grand-père, a été élu député à l'âge de vingt et un ans et « où l'aïeul a passé, passera le petit-fils $\gg_{82}$ L'Événement fait état de la ressemblance physique qui existe entre Laurier et Lavergne. « On le dit protégé par M. Laurier et cela se comprend, rien qu'à le voir », écrit un lecteur ${ }^{83}$ le journal lui-même ne trouve à Lavergne «d'autre mérite que de rajeunir les traits du premier ministre $\gg{ }^{84}$ Mais l'organe du gouvernement voit dans ce qu'il nomme prudemment «cette intimité... avec le premier ministre du Dominion... une garantie d'influence» pour les électeurs de Montmagny. ${ }^{85}$

Les arguments ne demeurent pas tous d'ordre politique ou personnel: la boisson apparaît (à Taschereau qui lui reprochera

78 A deux reprises, Lavergne a publié le texte de ce billet à lui présenté par Ernest Roy et émanant de Fitzpatrick, selon Choquette (Un demisiècle..., 148). Il est intéressant de noter que, à chaque reprise, le texte diffère. Selon le texte de 1907 (Débats de la Chambre..., 1906-1907, II: col. 2565), il lui fallait déclarer «formellement que j'approuve les actes du cabinet actuel et que je suis candidat ministériel purement et simplement ». D'après le texte de 1935 (Trente Ans..., 102), il lui fallait déclarer «par les présentes que j'approuve tous les actes passés du gouvernement libéral, et m'engage à les approuver à l'avenir ».

79 Armand Lavergne, Un mot de réplique, Lettre ouverte au Quebec Chronicle, publiée dans Le Soleil, 27 février 1904: 4; La Presse, 18 février 1904: 4.

80 La Presse, 8 février 1904: 4.

81 James D. Stewart à Choquette, 6 février 1904, Papiers Laurier, vol. 303: 82105 .

82 Le Soleil, 15 février 1904: 4.

83 Un Ancien de Montmagny, op. cit.

84 L'Evénement, 6 février 1904: 4.

85 Le Soleil, 15 février 1904: 4. 
plus tard d'avoir été élu à la faveur d'arguments ... liquides, Lavergne répliquera que «cela n'est pas impossible, attendu que tous vos chefs étaient là $\gg)^{86}$ et les conservateurs affirment que les libéraux ont dépensé $\$ 20,000.00$ pour donner la victoire à Lavergne. ${ }^{87}$ Car, quels que soient les procédés, Lavergne l'emporte par 328 voix et Laurier lui télégraphie: «Mille félicitations. Tu as remporté la plus belle victoire dans toutes les élections qui ont eu lieu hier. $\gg^{88}$

Assurément, on est en présence d'une belle victoire pour un jeune homme qui aura ses vingt-quatre ans quelques jours après son élection et que ses adversaires comparaient à l'Aiglon ! Mais la célébration de ce triomphe allait immédiatement ramener sur le tapis la question nationaliste, car plusieurs journaux attribuent à la Ligue la victoire dans Montmagny, seul comté où la majorité libérale ait augmenté $; 9$ de plus Tarte reconnaît en Bourassa et Lavergne des hommes «qui font partie d'un groupe qui constitue pour ainsi dire un tiers-parti $\gg .90$ Or voilà que la Ligue convoque une assemblée publique au Théâtre National de Montréal afin d'exposer sa doctrine, d'annoncer la toute prochaine parution de son hebdomadaire, et afin, également, de fêter le nouvel élu de Montmagny. Mais le juge et madame Lavergne mettent tout en œuvre pour empêcher Armand d'assister à cette assemblée ou, à tout le moins, d'y prendre la parole. Ils n'y réussissent pas parce que, écrit Joseph Lavergne à Rodolphe Boudreau, «il l'avait promis à Bourassa en reconnaissance de ce que ce dernier lui avait beaucoup aidé pendant l'élection et craignait de passer pour ingrat et lâche ». Et il ajoute: «Je voudrais de toutes manières détourner Armand de cette maudite Ligue... je veux qu'Armand soit un ministériel à tous crins. $»^{91}$ Mais

${ }^{86}$ Le Devoir, 23 février 1911.

87 L'Evénement, 17 février 1904: 1. $80465 \mathrm{c}$.

${ }^{88}$ Laurier à Lavergne, 17 février 1904, Papiers Laurier, vol. 297:

89 Le Nationaliste, 13 mars 1904: 2.

90 La Presse, 17 février 1904: 2.

91 Joseph Lavergne à Rodolphe Boudreau, 24 février 1904, Papiers Laurier, vol. $306: 82856$. 
le bon juge est déçu dans ses espoirs: Armand participe à l'assemblée du 21 février. Bourassa expose longuement le programme nationaliste et Lavergne proclame son anti-impérialisme, ajoutant que «si la Ligue Nationaliste n'était pas encore un parti politique, cela pourrait bien arriver avant longtemps $\gg .^{92}$ Le lendemain, il se rend à Ottawa et rencontre Laurier, sans toutefois oser lui demander de le présenter à la Chambre. ${ }^{93}$ Néanmoins, c'est aux bras de Laurier et de son oncle Louis qu'Armand Lavergne ${ }^{94}$ fera son entrée à la Chambre des Communes, ${ }^{95}$ le même jour qu'Ernest Lapointe. L'un et l'autre devaient, pendant longtemps, faire parler d'eux.

L'Université d'Ottawa.

MARC LA TERReUR

92 La Presse, 22 février 1904: 9.

93 Joseph Lavergne à Rodolphe Boudreau, 24 février 1904, Papiers Laurier, vol. 306: 82856 .

${ }^{94}$ On a pu remarquer que j'emploie l'orthographe Lavergne et La Vergne; en cela, je me conforme à l'orthographe de Lavergne lui-même qui n'écrivait pas son nom en deux mots au début de sa carrière.

95 Débats de la Chambre des Communes, session 1904, I: col. 6. 